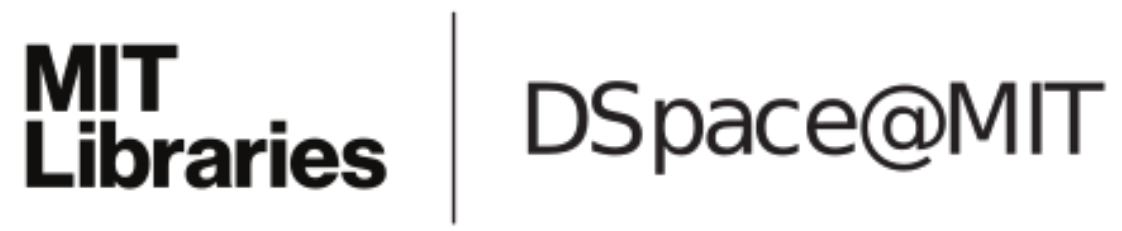

\author{
MIT Open Access Articles
}

\section{Atlas Generation for Subcortical and Ventricular Structures With Its Applications in Shape Analysis}

The MIT Faculty has made this article openly available. Please share how this access benefits you. Your story matters.

Citation: Anqi Qiu et al. "Atlas Generation for Subcortical and Ventricular Structures With Its Applications in Shape Analysis." Image Processing, IEEE Transactions on 19.6 (2010): 1539-1547. (c) Copyright 2010 IEEE

As Published: http://dx.doi.org/10.1109/tip.2010.2042099

Publisher: Institute of Electrical and Electronics Engineers

Persistent URL: http://hdl.handle.net/1721.1/61967

Version: Final published version: final published article, as it appeared in a journal, conference proceedings, or other formally published context

Terms of Use: Article is made available in accordance with the publisher's policy and may be subject to US copyright law. Please refer to the publisher's site for terms of use. 


\title{
Atlas Generation for Subcortical and Ventricular Structures With Its Applications in Shape Analysis
}

\author{
Anqi Qiu, Timothy Brown, Bruce Fischl, Jun Ma, and Michael I. Miller
}

\begin{abstract}
Atlas-driven morphometric analysis has received great attention for studying anatomical shape variation across clinical populations in neuroimaging research as it provides a local coordinate representation for understanding the family of anatomic observations. We present a procedure for generating atlas of subcortical and ventricular structures, including amygdala, hippocampus, caudate, putamen, globus pallidus, thalamus, and lateral ventricles, using the large deformation diffeomorphic metric atlas generation algorithm. The atlas was built based on manually labeled volumes of $\mathbf{4 1}$ subjects randomly selected from the database of Open Access Series of Imaging Studies (OASIS, 10 young adults, 10 middle-age adults, 10 healthy elders, and 11 patients with dementia). We show that the estimated atlas is representative of the population in terms of its metric distance to each individual subject in the population. In the application of detecting shape variations, using the estimated atlas may potentially increase statistical power in identifying group shape difference when comparing with using a single subject atlas. In shape-based classification, the metric distances between subjects and each of within-class estimated atlases construct a shape feature space, which allows for performing a variety of classification algorithms to distinguish anatomies.
\end{abstract}

Index Terms-Brain atlas, diffeomorphic mapping, shape classification, shape comparison, subcortical structures.

Manuscript received January 28, 2009; revised January 06, 2010. First published February 02, 2010; current version published May 14, 2010. This work was supported by National University of Singapore Start-Up Grant R-397-000058-133, A*STAR SERC 082101 0025, and A*STAR SICS 09/1/1/001. Support of this work was also provided in part by the National Center for Research Resources (P41 RR15241, P41-RR14075, R01 RR16594-01A1, and the NCRR BIRN Morphometric Project BIRN002, U24 RR021382), the National Institute for Biomedical Imaging and Bioengineering (R01 EB001550, R01EB006758), the National Institute for Neurological Disorders and Stroke (R01 NS052585-01) as well as the Mental Illness and Neuroscience Discovery (MIND) Institute, and is part of the National Alliance for Medical Image Computing (NAMIC), funded by the National Institutes of Health through the NIH Roadmap for Medical Research, under Grant U54 EB005149. The associate editor coordinating the review of this manuscript and approving it for publication was Dr. John Kerekes.

A. Qiu is with the Division of Bioengineering, National University of Singapore, Singapore 117576, the Singapore Institute for Clinical Sciences, Agency for Science, Technology and Research, Singapore 117574, Singapore, and also with the Clinical Imaging Research Center, National University of Singapore, Singapore 117576 (e-mail: bieqa@ nus.edu.sg).

T. Brown is with the Center for Imaging Science, Johns Hopkins University, Baltimore, MD 21218 USA

B. Fischl is with the Athinoula A. Martinos Center for Biomedical Imaging, Massachusetts General Hosptial, Boston, MA 02129 USA and also with the Computer Science and Artificial Intelligence Laboratory, Massachusetts Institute of Technology, Cambridge, MA 02139 USA.

J. Ma is with the Department of Biomedical Engineering, Johns Hopkins University, Baltimore, MD 21218 USA.

M. I. Miller is with the Singapore Institute for Clinical Sciences, the Agency for Science, Technology and Research, Singapore 117574, Singapore and also with the Athinoula A. Martinos Center for Biomedical Imaging, Massachusetts General Hosptial, Boston, MA 02129 USA.

Color versions of one or more of the figures in this paper are available online at http://ieeexplore.ieee.org.

Digital Object Identifier 10.1109/TIP.2010.2042099

\section{INTRODUCTION}

B RAIN warping techniques have made it possible to compare anatomical structure and function of the brain in large populations. Most of these techniques are atlas-based approaches that have been successful in describing anatomical variations between a collection of anatomies and an atlas [1]-[11]. The atlas is often represented by a healthy control subject from the population being studied. The difficulties with this approach are that the atlas may not be truly representative of the population, particularly when severe neurodegenerative disorders or brain development are studied. Wide variation of the anatomy across subjects relative to the atlas may cause the failure of the mapping. Thus, one of the fundamental limitations of choosing the anatomy of a single subject as an atlas is the introduction of a statistical bias based on the arbitrary choice of the atlas anatomy.

Atlas generation has received a great deal of attention [2], [12]-[18]. One of the earliest brain atlases is the Talairach and Tournoux atlas that was generated by dissecting and photographing a post-mortem sample from a woman with a smaller than average cranium, with associated Brodmann's areas labeled [12]. Because of the variability in brain size across subjects, most individual brains must be considerably deformed to fit the small size of this atlas. Nonetheless, the Talairach and Tournoux atlas is invaluable in modern neuroimaging. As imaging techniques (e.g., MRI, PET/CT) have matured, this atlas has paved the way for more representative brain atlases including the MNI atlas from the Montreal Neurological Institute (MNI) [13] and later the ICBM atlas from the International Consortium for Brain Mapping (ICBM) [15]. Both MNI and ICBM atlases were created by averaging linearly aligned MRI volumes in a large population. They became the standard atlases in Statistical Parametric Mapping (SPM) [19] and FSL [20] and have been widely used in functional studies, voxel-based morphometry (VBM), and tensor-based morphometry (TBM) (e.g., [3], [4], [21]-[24]). Associated probabilistic atlases that quantify the probability of tissue labels at each location were built for robust and automatic tissue segmentation as well [25], [26].

In recent years, researchers have developed more sophisticated atlas generation techniques based on nonlinear registration methods, in particular large deformation diffeomorphic mappings, to construct atlases with more detailed anatomical information than the MNI and ICBM for the purpose of the anatomical shape analysis [2], [14]. More recently, Trouvé et al. [27], [28] have developed a probabilistic framework coupled to the expectation-maximization (EM) algorithm for generating 
atlases from populations which have the property that the atlas is metrically centered via the diffeomorphic mapping [29].

In this paper, we focus on generating the atlas of an array of subcortical and ventricular structures, including the lateral ventricles, hippocampus, amygdala, caudate, putamen, globus pallidus, and thalamus, using large deformation diffeomorphism metric atlas generation approach. The atlas was estimated based on the 41 hand-labeled images that were selected from the database of Open Access Series of Imaging Studies (10 young adults, 10 middle-age adults, 10 healthy elders, and 11 patients with dementia). We anticipate that the estimated atlas will be useful in the shape difference detection and classification of subcortical and ventricular structures in a variety of neurodegenerate diseases and healthy aging.

\section{METHODS}

\section{A. Diffeomorphism Metric for Anatomical Shapes}

In the setting of diffeomorphic metric mapping, the set of anatomical shapes are placed into a metric shape space. Assume that the shape is generated one from the other via a flow of diffeomorphisms, solutions of ordinary differential equations $\dot{\phi}_{t}=v_{t}\left(\phi_{t}\right), t \in[0,1]$. The flow starts from the identity map, $\phi_{0}=\mathrm{id}$, and is associated with velocity vector fields $v_{t}, t \in$ $[0,1]$. We define a metric distance between target shape $I_{\text {targ }}$ and atlas shape $I_{\text {atlas }}$ as the length of the geodesic curves $\phi_{t} \cdot I_{\text {atlas }}$, $t \in[0,1]$ through the shape space such that $\phi_{1} \cdot I_{\text {atlas }}=I_{\text {targ }}$ at time $t=1$. For instance, in the image case, the group action is taken as $\phi_{1} \cdot I_{\text {atlas }}=I_{\text {atlas }} \circ \phi_{1}^{-1}$. These geodesics $\phi_{t} \cdot I_{\text {atlas }}, t \in[0,1]$ are generalizations of simple finite dimensional curves. The metric between two shapes $I_{\text {atlas }}, I_{\mathrm{targ}}$ is determined by the integrated norm $\left\|v_{t}\right\|_{V}$ of the vector field generating the transformation, where $v_{t} \in V$, a smooth Hilbert space with kernel $K$ and norm $\|\cdot\|_{V}$. To ensure solutions are diffeomorphisms, $V$ must be a space of smooth vector fields [30], [31]. Equivalently, the metric distance can be computed through $m_{t}$, termed the momentum, a linear transformation of $v_{t}$ defined by the kernel, $K: v_{t} \rightarrow m_{t}=K^{-1} v_{t}$ according to

$$
\begin{aligned}
\rho\left(I_{\text {atlas }}, I_{\text {targ }}\right)^{2} & =\inf _{v: \dot{\phi}_{t}=v_{t}\left(\phi_{t}\right), \phi_{0}=\mathrm{id}} \int_{0}^{1}\left\|v_{t}\right\|_{V}^{2} d t \\
& =\inf _{m_{t}: \dot{\phi}_{t}=K m_{t}\left(\phi_{t}\right), \phi_{0}=\mathrm{id}} \int_{0}^{1}\left\langle m_{t}, K m_{t}\right\rangle_{2} d t
\end{aligned}
$$

such that $\phi_{1} \cdot I_{\text {atlas }}=I_{\text {targ }}$. The Euler equation associated to (1) indicates that the momentum $m_{t}$ along the geodesic $\phi_{t}$ is conserved [29], [32], implying the initial momentum $m_{0}$ encodes the geodesic connecting $I_{\text {atlas }}$ and $I_{\text {targ }}$ according to

$$
m_{t}=\left|d \phi_{t}^{-1}\right|\left(d \phi_{t}^{-1}\right)^{*} m_{0} \circ \phi_{t}^{-1}
$$

where $d$ is the Jacobian matrix. This reduces the problem of studying shapes of a population in a nonlinear diffeomorphic metric space to a problem of studying the initial momenta in a linear space as has been done for landmarks in [33]. It thus forms the basis for our random orbit model on shapes below.

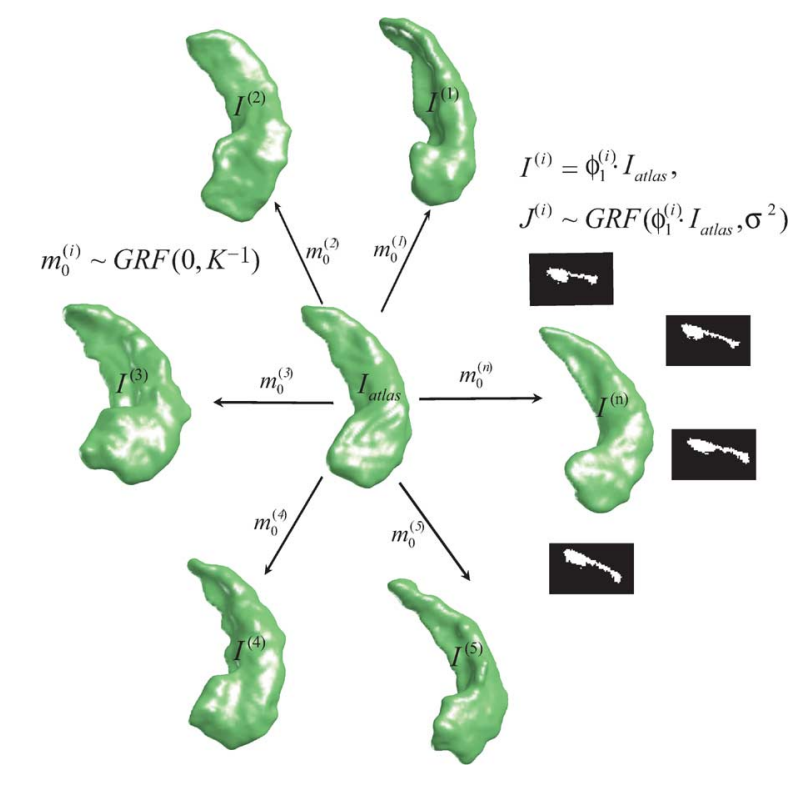

Fig. 1. Random orbit model includes two parts. The first part models initial momenta $m_{0}^{(i)}$ generating shape $I^{(i)}$ in the orbit of the atlas $I_{\text {atlas }}$ as Gaussian random field (GRF) with zero mean and covariance $K^{-1}$. The second part models an observed image $J^{(i)}$ as Gaussian random field with mean of shape $I^{(i)}=\phi_{1}^{(i)} \cdot I_{\text {atlas }}$ at the atlas orbit and variance $\sigma^{2}$.

\section{B. Random Orbit Shape Model}

We take as elements anatomical configurations $I \in \mathcal{I}$, functions indexed over $X \subset \mathcal{R}^{3}, I(x), x \in X$. We assume the orbit is generated from an atlas $I_{\text {atlas }} \in \mathcal{I}$. The atlas is unknown and must be estimated. All elements $I \in \mathcal{I}$ are generated by the flow of diffeomorphisms from the atlas for some $\phi_{t}, I=\phi_{1} \cdot I_{\text {atlas }}$, where $\phi_{t}$ is an element of the space of diffeomorphisms. We thus would like to estimate $I_{\text {atlas }}$ from a set of observed objects in $\mathcal{I}, J^{(1)}, J^{(2)}, \ldots, J^{(n)}$.

As illustrated in Fig. 1, our random model includes two parts. The first part of the random model assumes the anatomies $I^{(i)} \in$ $\mathcal{I}, i=1,2, \ldots, n$, are generated via geodesic flows of the diffeomorphism equation $\dot{\phi}_{t}^{(i)}=v_{t}^{(i)}\left(\phi_{t}^{(i)}\right)=K m_{t}^{(i)}\left(\phi_{t}^{(i)}\right), t \in$ $[0,1]$ from $I_{\text {atlas }}$, so that the conservation equation holds and the flow satisfies the conservations of the momentum equation (2). Thus, when $m_{0}^{(i)}, i=1,2, \cdots, n$ are considered as hidden variables, our probability law on $I^{(i)} \in \mathcal{I}$ is induced via the random law on the initial momenta $m_{0}^{(i)}$ in a linear space, which we shall model as independent and identically distributed Gaussian random fields (GRF) with zero mean and covariance matrix $K^{-1} / \lambda$, where $\lambda$ is constant and $K$ is the kernel as given in (1). The second part of the random model is the observable data $J^{(i)}$, the medical images. We assume the $J^{(i)}$ are conditional Gaussian random fields with mean fields $I^{(i)}=\phi_{1}^{(i)} \cdot I_{\text {atlas }}$ and variance $\sigma^{2}$. The goal is to estimate the atlas $I_{\text {atlas }}$ and $\sigma^{2}$ from the set of observables $J^{(1)}, J^{(2)}, \ldots, J^{(n)}$.

\section{EM Algorithm for Template Construction}

Given measured anatomical data sets $J^{(i)}, i=1, \ldots, n$, with unknown mean field $I^{(i)}=\phi_{t}^{(i)} \cdot I_{\text {atlas, }}$, our goal is to estimate the unknown atlas $I_{\text {atlas }}$ and $\sigma^{2}$. To solve for the unknown atlas, an ancillary "hyperatlas," $I_{0}$, is introduced so that our atlas is generated from it via the flow of diffeomorphisms of $\phi_{t}$ such that $I_{\text {atlas }}=\phi_{1} \cdot I_{0}$. Its associated 
momentum is denoted as $m_{0}$. We use the Bayesian strategy to estimate initial momentum $m_{0}$ from the set of observations $J^{(i)}, i=1, \ldots, n$ by computing the maximum a posteriori (MAP) of $f\left(m_{0}, \sigma^{2} \mid J^{(1)}, J^{(2)}, \cdots, J^{(n)}\right)$. To estimate $f\left(m_{0}, \sigma^{2} \mid J^{(1)}, J^{(2)}, \ldots, J^{(n)}\right)$, we include $m_{0}^{(1)}, \cdots, m_{0}^{(n)}$ associated with $\phi_{1}^{(i)}, i=1,2, \ldots, n$ as hidden variables. Thus, the log-likelihood of the complete data $\left(m_{0}, m_{0}^{(i)}, J^{(i)}\right.$, $i=1,2, \ldots, n)$ can be written as in (3), shown at the bottom of the page, where $I_{0}$ is the hyperatlas and $J^{(i)}$ is the observation of $i$ th subject. The paired $\left(\phi_{t}, m_{0}\right)$ and $\left(\phi_{t}^{(i)}, m_{0}^{(i)}\right)$ satisfy the geodesic shooting equation [32]. $K_{\pi}^{-1}$ and $K^{-1}$ are covariance matrices of $m_{0}$ and $m_{0}^{(i)}$ that are known and correspond to the kernel of Hilbert space of velocity fields. $D\left(\phi_{1}^{(i)} \cdot \phi_{1} \cdot I_{0}, J^{(i)}\right)$ quantifies the similarity between the $i$ th subject and deformed atlas $\phi_{1}^{(i)} \cdot \phi_{1} \cdot I_{0}$, which can be adapted to landmarks, images, curves, surface, and tensors [34]-[41]. $\sigma^{2}$ estimates the variance of the difference between $\phi_{1}^{(i)} \cdot \phi_{1} \cdot I_{0}$ and $J^{(i)}$ among $n$ subjects.

The above formulation has been solved for the image case using the expectation-maximization (EM) algorithm [28], where we define

$$
D\left(\phi_{1}^{(i)} \cdot \phi_{1} \cdot I_{0}, J^{(i)}\right)=\left\|I_{0} \circ \phi_{1}^{-1} \circ \phi_{1}^{(i)-1}-J^{(i)}\right\|^{2} .
$$

The E-step computes the expectation of the the complete data log-likelihood given the old atlas $m_{0}^{\text {old }}$ and variance $\sigma^{2^{\text {old }}}$ (see (4), shown at the bottom of the page). The M-step generates the new atlas by maximizing the Q-function with respect to $m_{0}, \sigma^{2}$, giving the update equation

$$
\begin{aligned}
m_{0}^{\text {new }}, \sigma^{2 \text { new }}= & \arg \max Q\left(m_{0}, \sigma^{2} \mid m_{0}^{\text {old }}, \sigma^{2^{\text {old }}}\right) \\
= & \arg \min _{m_{0}, \sigma^{2}} \lambda\left\langle m_{0}, K_{\pi} m_{0}\right\rangle_{2} \\
& +\sum_{i=1}^{n} E\left\{\frac{1}{\sigma^{2}}\left\|I_{0} \circ \phi_{1}^{-1} \circ \phi_{1}^{(i)-1}-J^{(i)}\right\|^{2}\right. \\
& \left.+n \log \sigma_{0}^{\text {old }}, \sigma^{2 \text { old }}, J^{(1)}, \ldots, J^{(n)}, I_{0}\right\}
\end{aligned}
$$

where we have used the fact that the conditional expectation of $\left\langle m_{0}^{(i)}, K m_{0}^{(i)}\right\rangle_{2}$ is constant. We solve $\sigma^{2}$ and $m_{0}$ by separating the procedure for updating $\sigma^{2}$ using the current value of $m_{0}$, and then optimizing $m_{0}$ using the updated value of $\sigma^{2}$. Thus, it yields

$$
\begin{aligned}
\sigma^{2 \text { new }}= & \frac{1}{n} \sum_{i=1}^{n}\left\|I_{0} \circ \phi_{1}^{-1 \text { old }} \circ \phi_{1}^{(i)-1}-J^{(i)}\right\|^{2} \\
m_{0}^{\text {new }}= & \arg \min _{m_{0}} \lambda\left\langle m_{0}, K_{\pi} m_{0}\right\rangle_{2} \\
& +\frac{1}{\sigma^{2 \text { new }}}\left\|\left(\bar{I}_{0}-I_{0} \circ \phi_{1}^{-1}\right) \sqrt{\alpha}\right\|^{2}
\end{aligned}
$$

where $\alpha=\sum_{i=1}^{n}\left|d \phi_{1}^{(i)}\right|$ is a weighted image volume to control the contribution of the image matching errors to the total cost at each voxel level. $\left|d \phi_{1}^{(i)}\right|$ is the Jacobian determinant of $\phi_{1}^{(i)}$. The mean image, $\bar{I}_{0}$, is computed in the form of

$$
\bar{I}_{0}=\frac{\sum_{i=1}^{n} J^{(i)} \circ \phi_{1}^{(i)}\left|d \phi_{1}^{(i)}\right|}{\sum_{i=1}^{n}\left|d \phi_{1}^{(i)}\right|} .
$$

The variational problem listed in (7) is referred as "modified LDDMM-image mapping" where the weight $\sqrt{\alpha}$ is introduced.

We briefly describe the steps involved in the estimation. In the first iteration, the hyperatlas is considered as the initial atlas where the corresponding $m_{0}=0$. The LDDMM-image mapping algorithm [39] is applied to register the current atlas to each individual subject and estimate each of $m_{t}^{(i)}$ and $\phi_{t}^{(i)}$. Thus, we can compute $\bar{I}_{0}$ and $\alpha$ based on $m_{t}^{(i)}$ and $\phi_{t}^{(i)}$. We then update $\sigma^{2}$ according to (6). The final step of each iteration, $m_{t}$ and $\phi_{t}$ connecting the hyperatlas to $\bar{I}_{0}$ is computed using a modified LDDMM-image mapping algorithm as given in (7). The new atlas is updated by $I_{0} \circ \phi_{1}^{-1}$. After each iteration, the new atlas moves close to the center of the metric space. This computation is repeated until the atlas converges.

\section{Atlas Generation Process}

Subjects and Image Acquisition: In the atlas generation procedure, 41 subjects with ten young adults ( 4 males and 6 females, age: $21.3 \pm 1.57), 10$ middle-aged adults ( 5 males and

$$
\begin{aligned}
\log f\left(m_{0}, \sigma^{2}, m_{0}^{(1)}, \ldots, m_{0}^{(n)}, J^{(1)}, \ldots J^{(n)} \mid I_{0}\right)=-\lambda\left\langle m_{0}, K_{\pi} m_{0}\right\rangle_{2} & -\sum_{i=1}^{n}\left\{\left\langle m_{0}^{(i)}, K m_{0}^{(i)}\right\rangle_{2}+\frac{1}{\sigma^{2}} D\left(\phi_{1}^{(i)} \cdot \phi_{1} \cdot I_{0}, J^{(i)}\right)\right\}-n \log \sigma^{2}
\end{aligned}
$$

$$
\begin{aligned}
Q\left(m_{0}, \sigma^{2} \mid m_{0}^{\text {old }}, \sigma^{2^{\text {old }}}\right)= & E\left\{\log f\left(m_{0}, \sigma^{2}, m_{0}^{(1)}, \ldots, m_{0}^{(n)}, J^{(1)}, \ldots J^{(n)} \mid I_{0}\right) \mid m_{0}^{\text {old }}, \sigma^{2 \text { old }}, J^{(1)}, \ldots, J^{(n)}, I_{0}\right\} \\
= & -\lambda\left\langle m_{0}, K_{\pi} m_{0}\right\rangle_{2}-E\left\{\sum_{i=1}^{n}\left[\left\langle m_{0}^{(i)}, K m_{0}^{(i)}\right\rangle_{2}+\frac{1}{\sigma^{2}}\left\|I_{0} \circ \phi_{1}^{-1} \circ \phi_{1}^{(i)-1}-J^{(i)}\right\|^{2}\right]\right. \\
& \left.+n \log \sigma^{2} \mid m_{0}^{\text {old }}, \sigma^{2 \text { old }}, J^{(1)}, J^{(2)}, \ldots, J^{(n)}, I_{0}\right\}
\end{aligned}
$$


5 females, age: $49.8 \pm 5.79$ ), 10 elders ( 5 males and 5 females, age: $73.6 \pm 7.05)$, and 11 patients with dementia ( 5 males and 6 females, age: $77.2 \pm 5.64$ ) were selected based on demographic information (gender, age, and diagnosis) from the database of Open Access Series of Imaging Studies (OASIS, http://www. oasis-brains.org). These subjects were also used to generate a probabilistic atlas for the subcortical segmentation in FreeSurfer [25].

All subjects were recruited in accordance with guidelines of the Washington University Human Studies Committee. All imaging was conducted at $1.5 \mathrm{~T}$ (Siemens Vision scanner, Erlangen Germany). Head movement was minimized by cushioning and a thermoplastic face mask. Three or four T1- weighted MP-RAGE [42] scans were acquired in each subject. MP-RAGE parameters were empirically optimized for gray-white contrast $(\mathrm{TR}=9.7 \mathrm{~ms}$, $\mathrm{TE}=4 \mathrm{~ms}$, flip angle $=10^{\circ}$, inversion time $(\mathrm{TI})=20 \mathrm{~ms}$, delay time $(\mathrm{TD})=200 \mathrm{~ms}, 256 \times 256(1 \times 1 \mathrm{~mm})$ in-plane resolution, 128 1.25-mm slices without gaps). The MP-RAGE data were averaged offline (with correction for head movement) to increase the contrast to noise ratio in all procedures involving manual tracing, segmentation, and measurement of normalized whole brain volume and were interpolated into isotropic voxels with resolution of $1 \mathrm{~mm} \times 1 \mathrm{~mm} \times 1 \mathrm{~mm}$.

Manual Segmentation: The averaged MRI scans were manually labeled as gray matter, white matter, ventricles (lateral and inferior lateral ventricles), and six subcortical structures (amygdala, hippocampus, thalamus, caudate, globus pallidus, putamen). The manual labeling was done at Massachusetts General Hospital and has been used as a training set for automatically segmenting subcortical and ventricular structures in FreeSurfer [25], [43]. The test-retest reliability of the manual segmentation procedure was assessed in a study in which each of five users labeled a single test image. The inter-rater reliability was quantified by percent volume overlap and volume difference (see details in [25]). The labeling masks that include lateral ventricles and the six subcortical structures were created for each subject based on the manual segmentation.

Rigid Alignment: One subject was randomly selected as the hyperatlas that was considered as the initial atlas. We rigidly aligned each individual labeling mask volume to the initial atlas using the cross-correlation criterion [44].

LDDMM Mapping and Template Estimation: For each individual structure, we applied the atlas generation algorithm described in Section II-C to rigidly aligned binary mask of the structure in these 41 subjects. During each iteration, the current atlas was deformed to all others using LDDMM-image mapping. $\sigma^{2}$ was estimated by taking the mean square of the difference between the deformed atlas and the images of all subjects. Then, a mean image was computed by averaging all transformed subject images weighted by their corresponding Jacobian determinant. The final step of each iteration, the diffeomorphic geodesic connecting the hyperatlas to the average image was computed using a modified LDDMM-image mapping algorithm. The new atlas was updated as the deformed hyperatlas. The stopping criteria were based on the rate of the change of averaged metric distance among all other 40 subjects.

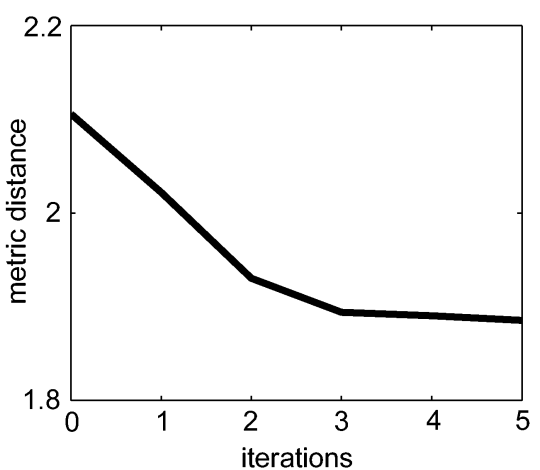

Fig. 2. Figure plots the averaged metric distances over 40 left hippocampi at each iteration.

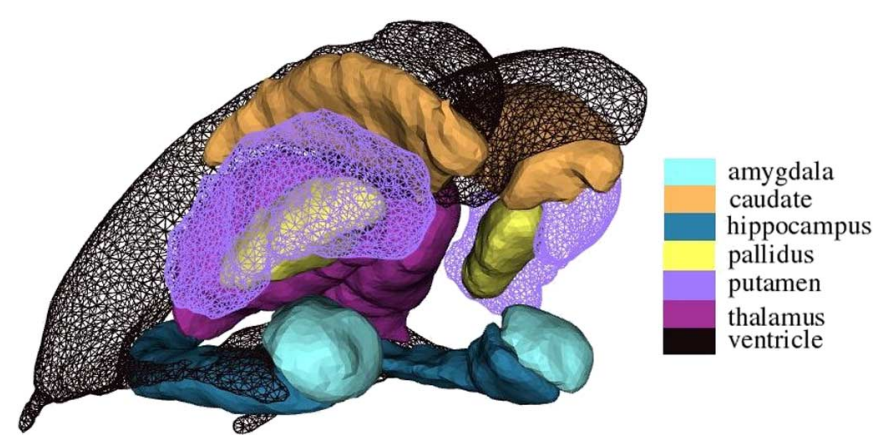

Fig. 3. Left and right atlas structures are shown in the surface representation. The structures include the lateral and inferior lateral ventricles, hippocampus, amygdala, thalamus, caudate, putamen, and globus pallidus.

Five iterations were taken in our analysis. Fig. 2 illustrates an example of the left hippocampal metric distance in each iteration. We replicated this procedure for each individual structure so that the volume representation of the atlas structures was created. The surface representation of the atlas structures was generated by isocontouring the volume.

Fig. 3 illustrates the estimated atlas in its surface representation, including both left and right sides. Both volume and surface representations of the atlas are now available online (see http://www.bioeng.nus.edu.sg/cfa/atlas/index.html). Fig. 3 color codes each structure as given in the legend. The estimated atlas for each individual structure has the property of smoothness and correct topology.

\section{RESULTS}

We will first present the usage of the estimated atlas for classifying populations based on the metric distances of subjects to the atlas. We will then discuss an advantage of the estimated atlas relative to a single subject atlas in shape analysis.

\section{A. Atlas Centered Classification}

By the construction of the diffeomorphic atlas estimation algorithm, the metric distance between the initial atlas (hyperatlas) and the manually labeled training subjects is larger than the one between the estimated atlas and these training subjects. Fig. 4(a) and (b) illustrates the hyperatlas and estimated atlas of the right side. Six subjects randomly selected from the 41 training subjects are shown in Fig. 4(c)-(h). The first number 


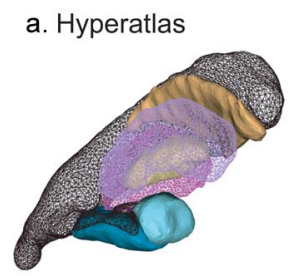

b. Estimated Atlas

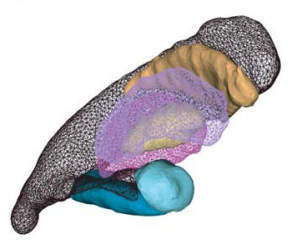

$$
\text { C. }
$$

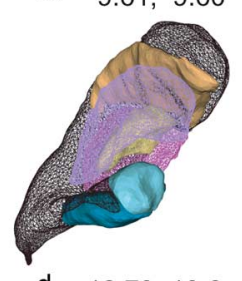

d. $12.73,10.6$

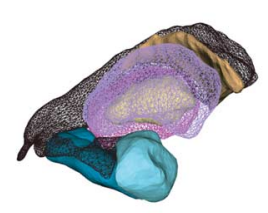

e. $10.33,9.33$

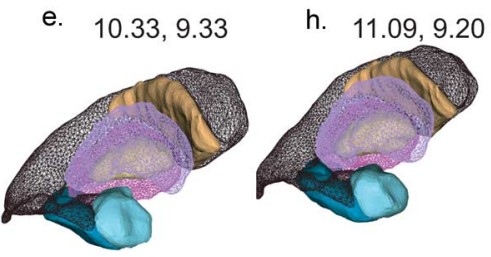

f. $11.86,9.82$

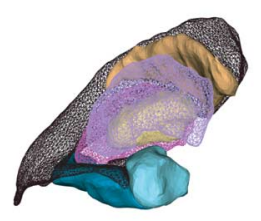

g.

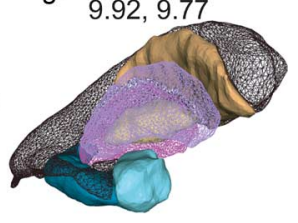

h. $11.09,9.20$
Fig. 4. Panels (a) and (b) respectively show the initial hyperatlas and estimated atlas of the right-side structures. Panels (c)-(h) illustrate the subcortical structures of six subjects. On the top of each panel, the first number is the metric distance between the hyperatlas and the subject summed over all seven structures. The second number is the metric distance between the estimated atlas and the subject. All structures on each panel are in the same color scheme as that shown in Fig. 3.
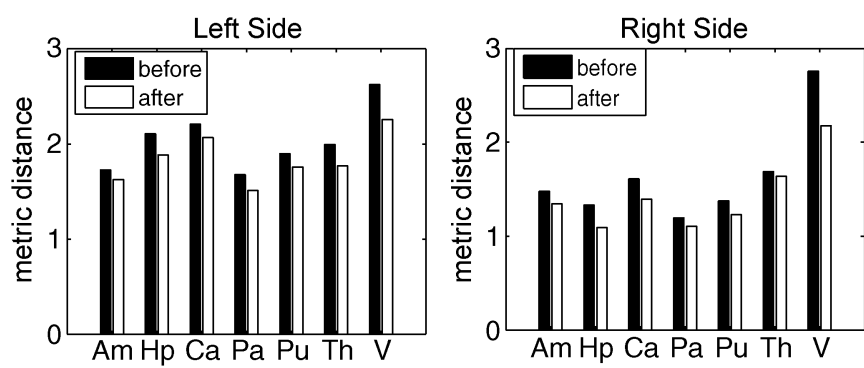

Fig. 5. Panels show the average metric distances over the group of subjects for various structures of the left and right sides, respectively. Dark and light bars represent the average metric distance before and after the atlas estimation. Consistently decreasing metric distance after the atlas generation procedure indicates that the estimated atlas is closer to each subject. Key: Am-Amygdala, $\mathrm{Hp}$-Hippocampus, $\mathrm{Ca}$-Caudate, $\mathrm{Pa}-$ Globus Pallidus, $\mathrm{Pu}$-Putamen, $\mathrm{V}$-lateral and inferior lateral ventricle.

on the top of each panel is the metric distance between the hyperatlas and the subject summed over the seven structures, while the second number is the metric distance between the estimated atlas and the subject. Fig. 5 summarizes the average metric distances over the 41 training subjects before and after the atlas estimation for each structure. These two figures show the consistent decrease of the metric distance in every structure of both left and right sides after the atlas generation, which suggests the estimated atlas is closer to each individual subject in the shape space. Thus, it indicates that the shapes of all subjects are roughly located in the orbit centered at the estimated atlas with the metric distance as radius in the shape space.

Based on the above property of the estimated atlas, we introduce a new shape classifier constructed based on the estimated (a)

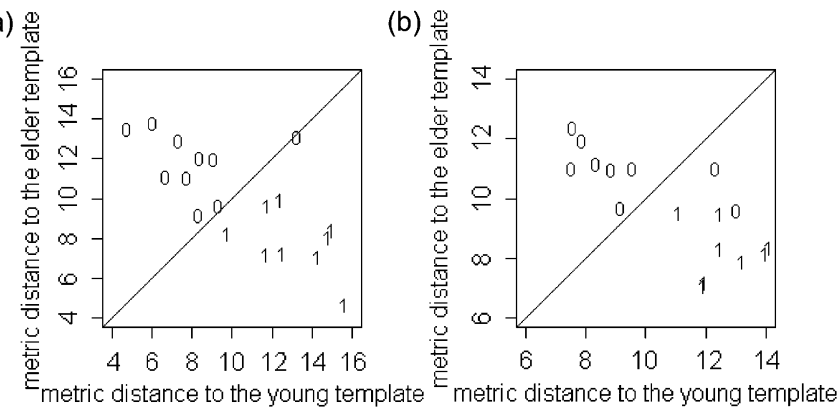

Fig. 6. Panels (a) and (b) respectively show the metric distance scatter plots of the training and test sets. "0" and "1" respectively represent true labels of young adults and elders.

atlases within each class and their metric distance to each individual subject. The basic idea of this shape classifier is similar to that of a traditional K-means classifier in computer vision. Instead of estimating the mean of each class during each iteration in the K-means classifier, we first estimate the mean shape of each class in the shape space using a training set. Then the metric distances of the within-class atlases to each individual subject construct a feature space that facilities classification algorithms to partition the feature space into discriminant regions. To illustrate how this classifier works, we gave an example to predict biological age of subjects based on their hippocampal shapes. The 20 hippocampi used in the previous section were selected as training data with two class labels (young adult, elderly). Additional 20 subjects were selected from the OASIS database as test data. Their gender and age were matched to those in the training data. We first estimated the atlases for each class of the training set using the procedure in Section II-D. The metric distances of all hippocampi to each atlas were computed via LDDMM-surface mapping [36], [45], as illustrated in Fig. 6(a) for the training data and (b) for the test data. The metric distances of each subject to each of the two estimated atlases were used as features in a simple classification algorithm, k-nearest neighbor. In the k-nearest neighbor classification, one neighbor was chosen. It can best discriminate the test data at $90 \%: 2$ out of 10 elders were labeled wrongly. The young adults can be well distinguished from the others. Discrimination will potentially be improved as increase in the sample size of the training set.

\section{B. Estimated Atlas Increases Statistical Power in Shape Comparison}

We demonstrate that the estimated atlas is superior to a singlesubject atlas for detecting group shape differences. To do so, hippocampal shape changes in the healthy aging were studied when the estimated atlas and two subjects' hippocampi were separately used as atlas in the shape comparison. 20 subjects were randomly selected from the OASIS database, including 10 young adults (5 males and 5 females) with mean age of 20.3 and 10 healthy elders (5 males and 5 females) with mean age of 72.0. The shape of the hippocampus in these subjects were represented by triangulated meshes.

As illustrated in Fig. 7, three hippocampi were used as atlas in the shape analysis. From the left panel to the right panel, Fig. 7 respectively shows the atlas estimated from the 41 training subjects and two single-subject hippocampi randomly chosen 


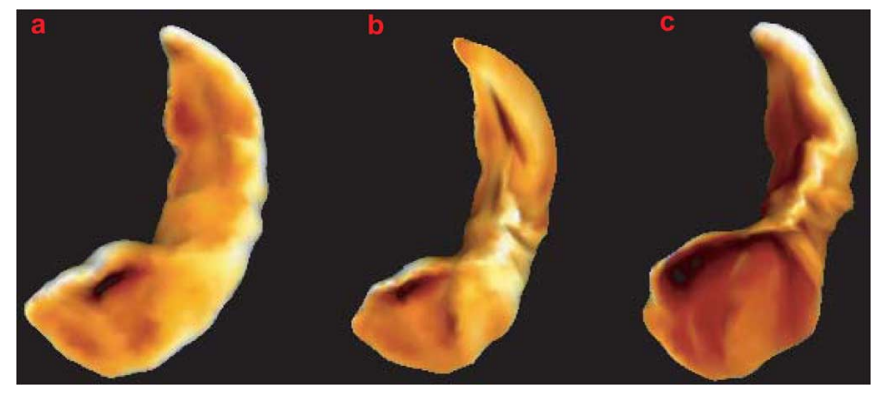

Fig. 7. Panels (a)-(c) respectively show the atlas estimated from the 41 training subjects and two single-subject hippocampi randomly chosen from the OASIS database. The hippocampal surfaces are colored by the mean curvature.

\section{Mapping Error Distance Maps}

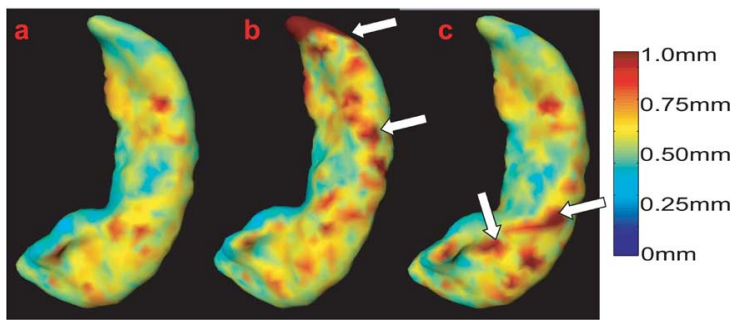

Fig. 8. Panels (a)-(c) respectively show the mapping error distance maps in the estimated hippocampal coordinates. Each panel shows the map associated with the atlas in the corresponding panel in Fig. 7.

from the OASIS database. We applied the LDDMM-surface mapping [36], [45] for separately registering each of the three atlases to the 20 hippocampi. Given an atlas, its mapping error map was computed as Euclidean distance between its deformed version and the subject's hippocampus. To visually compare the mapping errors when the three different atlases were used in the registration, Fig. 8 illustrates the average mapping error maps over the 20 subjects in the estimated atlas coordinates, while Fig. 9 shows the mean distribution of the mapping error distance. These two figures first suggest that the mapping errors are not uniformly distributed over the hippocampal surface in the order of less than MRI resolution of $1 \mathrm{~mm}$. Secondly, the estimated atlas gives the smallest mapping errors when compared with the two single-subject atlases. Kolmogorov-Smirnov tests further confirmed that the distribution of the mapping error distance using the estimated atlas is larger than those using single-subject atlases $(p<0.0001)$, i.e., more vertices on the surface with small error distances using the estimated atlas when compared with using single-subject atlases. Fig. 8(b) shows the single-subject atlas [Fig. 7(b)] has large mapping errors in the hippocampal tail and the superior body; Fig. 8(c) shows the single-subject atlases [Fig. 7(c)] has large mapping errors in the superior body near the head as pointed by white arrows.

For detecting hippocampal shape differences between young and elder adults, the Jacobian determinant of the LDDMM transformation was computed, and its logarithmic scale was used in the statistical testing. Fig. 10 shows the difference in the average Jacobian determinant map in the logarithmic scale between the groups of young adults and elders. From left to right, panels respectively show the results corresponding to the three atlases in Fig. 7. The color represents a ratio of the

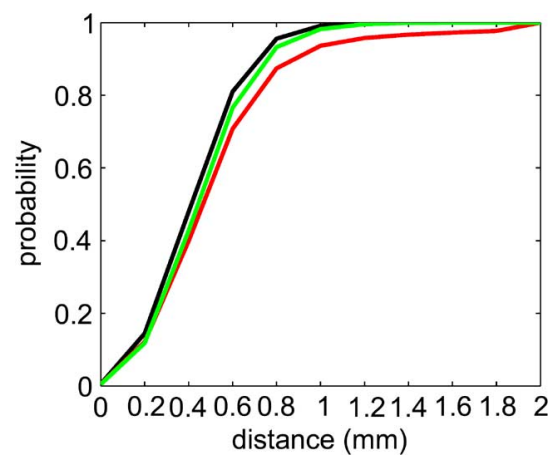

Fig. 9. Black, red, and green curves respectively correspond to the mean distribution of the mapping error distance among the 20 subjects when the estimated atlas and two single-subject atlases (Fig. 7) were used in mapping. Each curve shows the percentage of vertices on the atlas with mapping error distance less than $d \mathrm{~mm}$.

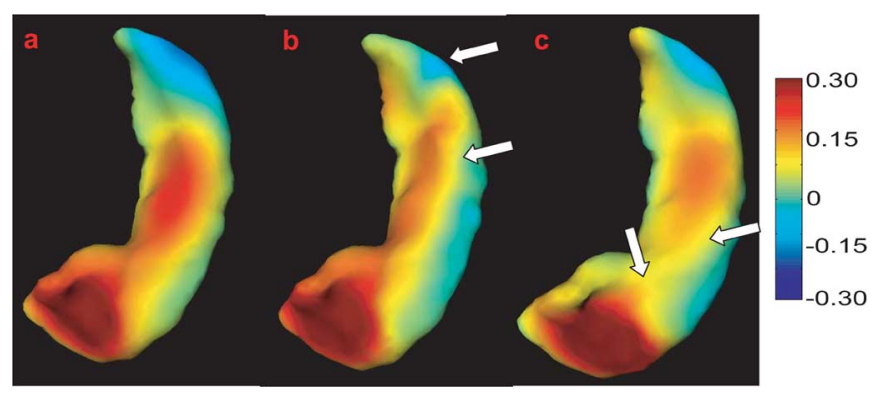

Fig. 10. Panels (a)-(c) show the difference in the average logarithmic scale of the Jacobian determinant between the groups of the young adults and elders when the atlases shown in Fig. 7 were used in shape analysis. The color scale represents a ratio of the average hippocampal volume in the group of the young adults to the one in the group of the elders in logarithmic scale. Arrows point at the regions with large mapping errors as shown in Fig. 8.

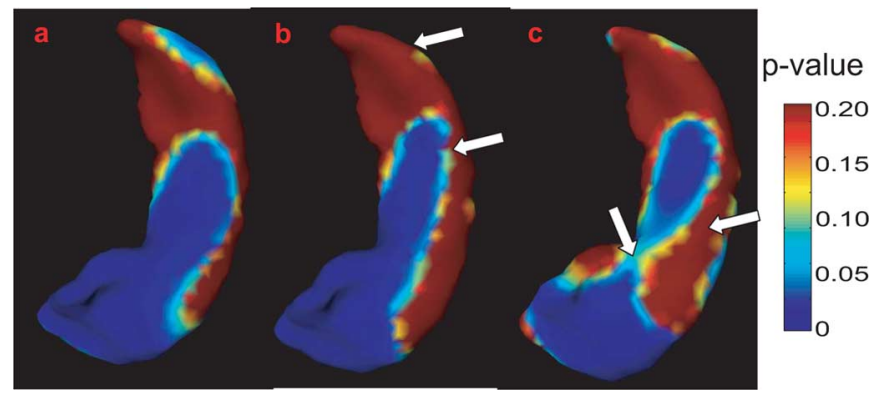

Fig. 11. Figure shows the p-value maps representing the shape difference between the groups of the young adults and elders. Each panel shows the map associated with the atlas in the corresponding panel in Fig. 7.

hippocampal volume in the group of the young adults to the one in the group of the elders in the logarithmic scale. Thus, when compared with the hippocampal shape in the young adults, red denotes the region where the hippocampus is compressed in the group of elders, while blue represents the region where the hippocampus is expanded in the group of elders. To statistically explore the shape difference between the two groups, at each vertex the logarithmic scale of the Jacobian determinant was modeled using linear regression with group information as independent variable and the estimated total intracranial volume (eTIV) [46] as covariate. Fig. 11 shows the p-value map associated with the grouping variable obtained from the linear regression model. Using the three different atlases, we 
were able to detect the large portion of the hippocampal head and a small patch of the lateral aspect of the hippocampal tail with p-value less than 0.05 . The comparison between panels (a) and (b) or (a) and (c) in Figs. 10 and 11 suggests that the statistical results from the estimated atlas and a single-subject atlas in the shape analysis are in close agreement. Out of total 1184 vertices on the surface, 719,622 , and 547 vertices have p-values less than 0.05 when respectively using the atlases shown in Fig. 7. Therefore, using the estimated atlas in the shape comparison detects a larger region with p-value less than 0.05 than using any of the single-subject atlases. This is partly due to larger mapping error or deformation variance in mapping the single subject atlas to the population. As illustrated in Fig. 8, the estimated hippocampal atlas gives the smallest mapping error [panel (a)] compared to the two single subject atlases [panels (b) and (c)]. The regions where the statistical power is lost (pointed by the arrows in Fig. 11) well correspond the regions with large mapping errors (pointed by the arrows in Fig. 8). We thus conclude that using the estimated atlas in shape analysis increases statistical power in detecting the shape differences between groups. Notice that a single-subject atlas may give equivalent statistical results as the estimated atlas if its shape is close to the estimated atlas in the shape space. Moreover, p-values shown in Fig. 11 are uncorrected for multiple comparisons.

\section{Discussion}

We created the atlas shapes of the subcortical and ventricular structures, including the lateral ventricles, hippocampus, amygdala, caudate, putamen, globus pallidus, and thalamus, using the large deformation diffeomorphic metric atlas generation algorithm [28]. The atlas was built based on the manually labeled image volumes. From the construction of the diffeomorphic atlas generation algorithm, the estimated atlas has a representative shape in the shape space in terms of its metric distances to each individual subject. We now make both volume and surface representations of the atlas available online to be accessed (http://www.bioeng.nus.edu.sg/cfa/atlas/index.html). We demonstrated the applications of this estimated atlas in both shape classification and shape comparison.

Effects of the Hyperatlas Choice: Mapping results from atlasbased brain mapping tools in general are atlas dependent. The level of the dependency is determined by the mapping accuracy. We would thus expect that our estimated atlas is dependent on the shape of the hyperatlas in a certain degree. Fig. 12 shows three hyperatlases and the estimated atlases using them, which indicates the relationship between the hyperatlas shape and estimated atlas shape. Nevertheless, the LDDMM mapping algorithms provide accurate registration [36], [47]. They thus result in the estimated atlases in similar shapes even though the hyperatlases are quite far from one to the other [Fig. 12(a)-(c)].

Comparisons With Other Atlas Estimation Approaches: The atlas estimation method in [14] is a simplified model of our approach when the estimated atlas is computed only using (8) without weights of the Jacobian determinant. This simplified model indicates that the estimated atlas is a linear combination of images, which blurs the boundary of the structures. Moreover, the image obtained from simple average is not guaranteed to be

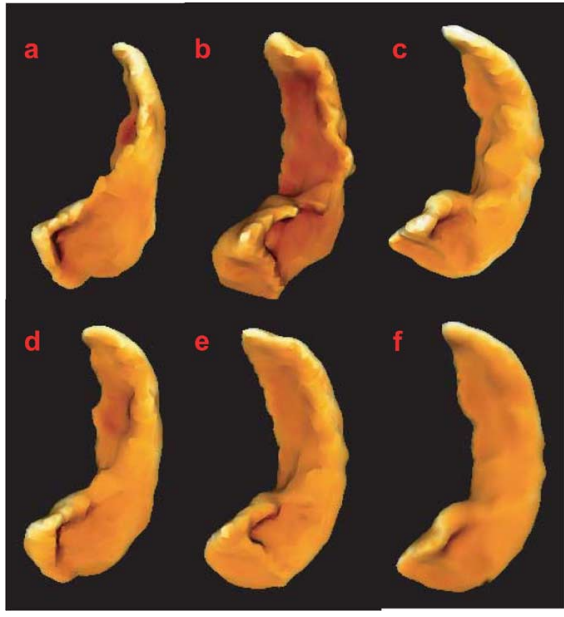

Fig. 12. Panels (a)-(c) are hyperatlases. Panels (d)-(f) show the estimated atlases using the hyperatlases in panels (a)-(c), respectively. The surface is colored based on its curvature.

in the same shape space as the initial atlas. In contrast, our atlas estimation model introduces the hyperatlas which the estimated atlas is generated from through the modified LDDMM mapping. Thus, the estimated atlas is in the orbit of the hyperatlas in the shape space and also the clear boundary of the hyperatlas is preserved (see comparison examples in [28]). Compared with the method in [2], our method is robust to shape outliers when they are used for the atlas generation. Avants and his colleagues generated an atlas via shooting an initial atlas by averaged initial velocity over a population. This guarantees that the estimated atlas is in the same shape space as the initial atlas. However, this shooting model does not consider the variance of the initial velocity. Thus, one shape outlier in the population may cause the failure in the atlas estimation. In contrast to Avants' approach, our atlas estimation built the covariance structure of the initial momentum in our probabilistic model, this makes the method robust to shape outliers. One advantage of the atlas estimation method in [2] is that the symmetrical diffeomorphic mapping algorithm was used to guarantee a unique geodesic path found in the mapping from the atlas to a target or from the target to the atlas while our LDDMM algorithm in practice may not necessarily satisfy this condition. Compared with other atlas generation approaches using nonlinear registration algorithms, such as one in [18], our method directly works on the diffeomorphic transformation after rigid motion is removed from all images. Guimond et al. [18] introduced a way to combine the affine transformation and elastic transformation together for the atlas generation. Similar to Joshi's method [14], Guimond's method also took average of deformed images, which may blur the boundary of the structures.

Shape Classification: To our best knowledge, the atlascentered classifier is the first shape classifier built based on within-class atlases and their diffeomorphic metric distances to each individual subject. In this paper, we provided a supervised approach when a training set with label information was known. This classifier applied the diffeomorphic atlas generation algorithm to estimate within-class atlas structure as a mean of each class in a diffeomorphic metric shape space from a training set. 
The metric distance of subjects to each of within-class atlases is thus considered as feature to best separate the feature space into discriminant regions. As a simple illustration, we showed the atlas-centered classifier can predict biological age of subjects at high accuracy rate and diagnosis of mild dementia at relatively low accuracy rate based on the hippocampal shapes. The improvement of the classification may be achieved when more hippocampal shapes of the elderly and patients with dementia are used to incorporate large shape variations in these two groups in the atlas estimation and sophisticated nonlinear classification algorithms can be considered. However, we focused on constructing a feature space using the class atlases. Thus, a simple classification approach, k-nearest neighbor, was applied in this study. To extend this classification approach, a nonsupervised classification scheme can be designed as well. The mean atlas of each class can be initialized as one of subjects in a dataset. Subjects can be labeled as the class whose atlas has the minimal metric distance to it. The atlas estimation procedure is then used to estimate a new atlas for each class. The above procedure can be repeated until no label is changed. Of course, this nonsupervised classification method will be costly in terms of the computation time. Nevertheless, the shape classification approach provided in this paper may motivate a new way to classify subjects based on their structural shapes and atlas shapes constructed from a population.

Shape Comparison: In the application of shape comparison, the estimated atlas reduces the average mapping error when comparing with a single subject atlas. Thus, the estimated atlas potentially increases statistical power in detecting shape difference across groups. We have integrated this subcortical and vetricular atlas with statistical analysis on multiple structure shapes into a shape analysis pipeline [48] for delineating subcortical and ventricular structures with correct topology and detecting anatomical connectivity among these subcortical and ventricular structures in the atlas coordinate on the basis of similar shape alterations associated with a disease. With this atlas, the shape analysis pipeline has been successfully used to assess the subcortical shape abnormalities in MCI and AD in 400 MRI scans shared through Alzheimer's Disease NeuroImaging Initiative (ADNI) [49].

Following the atlas generation procedure presented in this paper, we also created a shape atlas of the basal ganglia (caudate, putamen, globus pallidus) in healthy children aged from 7 to 12 years (http://www.bioeng.nus.edu.sg/cfa/atlas/index.html). This atlas can be also used to assess morphometric shape abnormalities of the basal ganglia in a variety of neurodevelopmental disorders with dysfunctions regulated by the basal ganglia, such as ADHD and Autism [50].

\section{REFERENCES}

[1] U. Grenander and M. I. Miller, "Computational anatomy: An emerging discipline," Q. Appl. Math., vol. 56, no. 4, pp. 617-694, 1998.

[2] B. Avants and J. C. Gee, "Geodesic estimation for large deformation anatomical shape and intensity averaging," NeuroImage, vol. 23, pp. $139-150,2004$.

[3] J. Ashburner, J. G. Csernansky, C. Davatzikos, N. C. Fox, G. B. Frisoni, and P. M. Thompson, "Computer-assisted imaging to assess brain structure in healthy and diseased brains," Lancet Neurol., vol. 2, pp. 79-88, 2003.

[4] J. Ashburner, "A fast diffeomorphic image registration algorithm," Neuroimage, vol. 38, pp. 95-113, 2007.
[5] L. G. Apostolova, R. A. Dutton, I. D. Dinov, K. M. Hayashi, A. W Toga, J. L. Cummings, and P. M. Thompson, "Conversion of mild cognitive impairment to alzheimer disease predicted by hippocampal atrophy maps," Arch. Neurol., vol. 63, pp. 693-699, 2006.

[6] L. G. Apostolova, I. D. Dinov, R. A. Dutton, K. M. Hayashi, A. W. Toga, J. L. Cummings, and P. M. Thompson, "3D comparison of hippocampal atrophy in amnestic mild cognitive impairment and alzheimer's disease," Brain, vol. 129, pp. 2867-2873, 2006.

[7] J. G. Csernansky, M. K. Schindler, N. R. Spliner, L. Wang, M. Gado, L. D. Selemon, D. Rastogi-Cruz, P. A. Posener, and M. I. Miller, "Abnormalities of thalamic volume and shape in schizophrenia," Amer. $J$. Psych., vol. 161, pp. 896-902, 2004.

[8] J. G. Csernansky, L. Wang, S. C. Joshi, J. T. Ratnanather, and M. I. Miller, "Computational anatomy and neuropsychiatric disease: Probabilistic assessment of variation and statistical inference of group difference, hemispheric asymmetry, and time-dependent change," NeuroImage, vol. 23, pp. S139-S150, 2004.

[9] T. Paus, A. Zijdenbos, K. Worsley, D. Collins, J. Blumenthal, J. Giedd, J. Rapoport, and A. Evans, "Structural maturation of neural pathways in children and adolescents: In vivo study," Science, vol. 283, pp. 1908-1911, 1999.

[10] P. M. Thompson, J. N. Giedd, R. P. Woods, D. MacDonald, A. C. Evans, and A. W. Toga, "Growth patterns in the developing brain detected by using continuum mechanical tensor maps," Nature, vol. 404, pp. 190-193, 2000.

[11] E. R. Sowell, B. S. Peterson, P. M. Thompson, S. E. Welcome, A. L. Henkenius, and A. W. Toga, "Mapping cortical change across the human life span," Nat. Neurosci., vol. 6, no. 3, pp. 309-315, 2003.

[12] J. Talairach and P. Tournoux, Co-Planar Stereotaxic Atlas of the Human Brain: 3-Dimensional Proportional System-An Approach to Cerebral Imaging. New York: Thieme, 1988.

[13] A. C. Evans, D. L. Collins, S. R. Mills, E. D. Brown, R. L. Kelly, and T. M. Peters, "3D statistical neuroanatomical models from 305 MRI volumes," in Proc. IEEE Nuclear Science Symp. and Medical Imaging Conf., 1993, pp. 1813-1817.

[14] S. C. Joshi, B. Davis, M. Jomier, and G. Gerig, "Unbiased diffeomorphic atlas construction for computational anatomy," NeuroImage, vol. 23, pp. 151-160, 2004.

[15] J. Mazziotta, A. Toga, A. Evans, P. Fox, J. Lancaster, K. Zilles, R. Woods, T. Paus, G. Simpson, B. Pike, C. Holmes, L. Collins, P. Thompson, D. MacDonald, M. Iacoboni, T. Schormann, K. Amunts, N. Palomero-Gallagher, S. Geyer, L. Parsons, K. Narr, N. Kabani, G. Le Goualher, D. Boomsma, T. Cannon, R. Kawashima, and B. Mazoyer, "A probabilistic atlas and reference system for the human brain: International consortium for brain mapping (ICBM)," Philos. Trans. R. Soc. London B: Biol. Sci., vol. 356, pp. 1293-1322, 2001.

[16] P. Lorenzena, M. Prastawaa, B. Davisa, G. Geriga, E. Bullitta, and S. Joshia, "Multi-modal image set registration and atlas formation," Med. Image Anal., vol. 10, pp. 440-451, 2006.

[17] B. Davis, P. Lorenzen, and S. Joshi, "Large deformation minimum mean squared error template estimation for computational anatomy," in Proc. IEEE Int. Symp. Biomed. Imaging: Macro to Nano, 2004, pp. $173-176$.

[18] A. Guimond, J. Meunier, and J.-P. Thirion, "Average brain models: A convergence study," Comput. Vision Image Underst., vol. 77, no. 2, pp. $192-210,2000$.

[19] K. J. Friston, J. T. Ashburner, S. J. Kiebel, T. F. Nichols, and W. D. Penny, Statistical Parametric Mapping: The Analysis of Functional Brain Images. Amsterdam, The Netherlands: Elsevier, 2007.

[20] S. Smith, M. Jenkinson, M. Woolrich, C. Beckmann, T. Behrens, H. Johansen-Berg, P. Bannister, M. D. Luca, I. Drobnjak, D. Flitney, R. Niazy, J. Saunders, J. Vickers, Y. Zhang, N. D. Stefano, J. Brady, and P. Matthews, "Advances in functional and structural MR image analysis and implementation as FSL," Neurolmage, vol. 23, pp. 208-219, 2004.

[21] J. Ashburner and K. J. Friston, "Voxel-based morphometry-The methods," NeuroImage, vol. 11, pp. 805-821, 2000.

[22] M. K. Chung, K. J. Worsley, S. Robbins, T. Paus, J. Taylor, J. Griedd, J. L. Rapoport, and A. C. Evans, "Deformation-based surface morphometry applied to gray matter deformation," NeuroImage, vol. 18, pp. 193-213, 2003.

[23] K. Friston, J. Ashburner, C. Frith, J.-B. Poline, J. D. Heather, and R. Frackowiak, "Spatial registration and normalization of images," Human Brain Mapping, vol. 2, pp. 165-189, 1995.

[24] C. Good, I. Johnsrude, J. Ashburner, R. Henson, K. Friston, and R. Frackowiak, "A voxel-based morphometric study of ageing in 465 normal adult human brains," NeuroImage, vol. 14, pp. 21-36, 2001.

[25] B. Fischl, D. H. Salat, E. Busa, M. Albert, M. Dieterich, C. Haselgrove, A. van der Kouwe, R. Killiany, D. Kennedy, S. Klaveness, A. Montillo, N. Makris, B. Rosen, and A. M. Dale, "Whole brain segmentation: Automated labeling of neuroanatomical structures in the human brain," Neuron, vol. 33, pp. 341-355, 2002. 
[26] J. Ashburner and K. J. Friston, "Unified segmentation," Neurolmage, vol. 26, pp. 839-851, 2005.

[27] S. Allassonniére, Y. Amit, and A. Trouvé, "Towards a coherent statistical framework for dense deformable template estimation," J. R. Stat. Soc. Series B, vol. 69, no. 1, pp. 3-29, 2007.

[28] J. Ma, M. I. Miller, A. Trouvé, and L. Younes, "Bayesian template estimation in computational anatomy," NeuroImage, vol. 42, pp. 252-261, 2008.

[29] M. I. Miller, A. Trouvé, and L. Younes, "On metrics and Euler-Lagrange equations of computational anatomy," Ann. Rev. Biomed. Eng., vol. 4, pp. 375-405, 2002.

[30] A. Trouvé, An infinite dimensional group approach for physics based models Tech. Rep., 1995 [Online]. Available: http://www.cis.jhu.edu

[31] P. Dupuis, U. Grenander, and M. I. Miller, "Variational problems on flows of diffeomorphisms for image matching," Q. Appl. Math., vol. 56, pp. 587-600, 1998.

[32] M. I. Miller, A. Trouvé, and L. Younes, "Geodesic shooting for computational anatomy," J. Math. Imaging Vision, vol. 24, pp. 209-228, 2006.

[33] M. Vaillant, M. I. Miller, L. Younes, and A. Trouvé, "Statistics on diffeomorphisms via tangent space representations," NeuroImage, vol. 23, pp. 161-169, 2004.

[34] A. Qiu, L. Younes, and M. I. Miller, "Intrinsic and extrinsic analysis in computational anatomy," Neuroimage, vol. 39, pp. 1803-1814, 2008.

[35] A. Qiu and M. I. Miller, "Cortical hemisphere registration via large deformation diffeomorphic metric curve mapping," in Proc. 10th Int. Conf. Medical Image Computing and Computer Assisted Intervention, 2007, vol. 10, pp. 186-193.

[36] M. Vaillant, A. Qiu, J. Glaunès, and M. I. Miller, "Diffeomorphic metric surface mapping in subregion of the superior temporal gyrus," NeuroImage, vol. 34, pp. 1149-1159, 2007.

[37] S. Joshi and M. I. Miller, "Landmark matching via large deformation diffeomorphisms," IEEE Trans. Image Process., vol. 9, no. 8, pp. 1357-1370, Aug. 2000.

[38] J. Glaunès, A. Trouvé, and L. Younes, "Diffeomorphic matching of distributions: A new approach for unlabelled point-sets and sub-manifolds matching," in Proc. IEEE Computer Society Conf. Computer Vision and Pattern Recognition, 2004, pp. 712-718.

[39] M. F. Beg, M. I. Miller, A. Trouvé, and L. Younes, "Computing large deformation metric mappings via geodesic flows of diffeomorphisms," Int. J. Comput. Vision, vol. 61, no. 2, pp. 139-157, Feb. 2005.

[40] Y. Cao, M. I. Miller, R. L. Winslow, and L. Younes, "Large deformation diffeomorphic metric mapping of fiber orientations," in Proc. 10th Int. Conf. Comput. Vision, Beijing, China, Oct. 2005, pp. 1379-1386.

[41] Y. Cao, M. Miller, R. Winslow, and L. Younes, "Large deformation diffeomorphic metric mapping of vector fields," IEEE Trans. Med. Imaging, vol. 24, pp. 1216-1230, 2005.

[42] J. P. Mugler and J. R. Brookeman, "Three-dimensional magnetizationprepared rapid gradient-echo imaging (3D MP RAGE)," Magn. Reson. Med., vol. 15, no. 9, pp. 152-157, Sep. 1990.

[43] B. Fischl, D. Salat, M. A. J. W. van der Kouwe, F. N. Ségonne, and A. Dale, "Sequence-independent segmentation of magnetic resonance images," NeuroImage, vol. 23, pp. S69-S84, 2004.

[44] M. Jenkinson and S. Smith, "A global optimisation method for robust affine registration of brain images," Med. Image Anal., vol. 5, pp. 143-156, 2001.

[45] M. Vaillant and J. Glaunès, "Surface matching via currents," in Information Processing in Medical Imaging. Berlin, Germany: Springer-Verlag, 2005, vol. 3565, Lecture Notes in Computer Science, pp. 381-392.

[46] R. L. Buckner, D. Head, J. Parker, A. F. Fotenos, D. Marcus, J. C. Morris, and A. Z. Snyder, "A unified approach for morphometric and functional data analysis in young, old, and demented adults using automated atlas-based head size normalization: Reliability and validation against manual measurement of total intracranial volume," $\mathrm{Neu}$ roimage, vol. 23, pp. 724-738, 2004.

[47] M. I. Miller, M. F. Beg, C. Ceritoglu, and C. Stark, "Increasing the power of functional maps of the medial temporal lobe by using large deformation diffeomorphic metric mapping," Proc. Nat. Acad. Sci., vol. 102 , pp. 9685-9690, 2005.

[48] A. Qiu and M. I. Miller, "Multi-structure network shape analysis via normal surface momentum maps," NeuroImage, vol. 42, no. 4, pp. 1430-1438, 2008

[49] A. Qiu, C. Fennema-Notestine, A. Dale, and M. Miller, Alzheimer's Disease Neuroimaging Initiative, "Regional shape abnormalities in mild cognitive impairment and Alzheimer's disease," Neuroimage, vol. 45, pp. 656-661, 2009.
[50] A. Qiu, D. Crocetti, M. Adler, M. Mahone, M. Deckla, M. I. Miller, and S. H. Mostofsy, "Basal ganglia volume and shape in children with ADHD," Amer. J. Psych., vol. 166, no. 1, pp. 74-82, 2009.

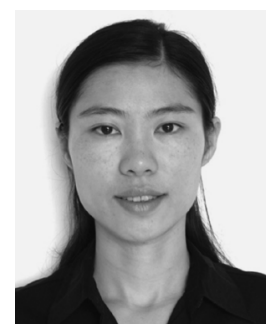

Anqi Qiu received the Ph.D. degree from The Johns Hopkins University, Baltimore, MD, in 2006.

She is currently an Assistant Professor at the National University of Singapore. She has been there since 2007. Her research focuses on multimodal brain magnetic resonance imaging analysis.

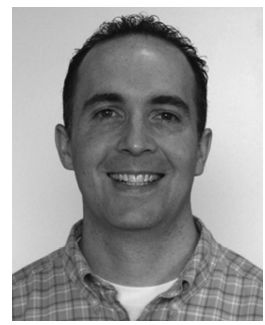

Timothy Brown is currently a Software Engineer at the Center for Imaging Science, Johns Hopkins University, Baltimore, MD.

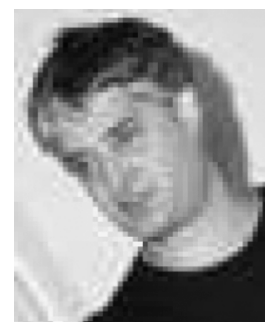

Bruce Fischl is currently an Associate Professor of radiology at Harvard Medical School and an Associate Neuroscientist at Massachusetts General Hospital, Boston. His current research involves the development of techniques for the automatic construction and utilization of geometrically accurate and topologically correct models of the human cerebral cortex.

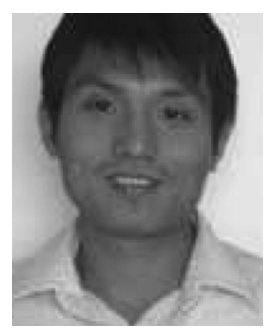

Jun Ma is currently a Graduate Student at the Center for Imaging Science, Johns Hopkins University, Baltimore, MD

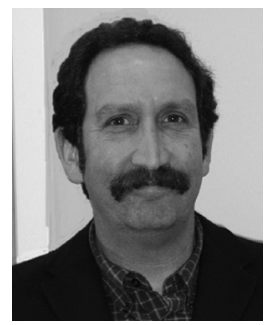

Michael I. Miller is currently a Professor at the Department of Biomedical Engineering and the Director of the Center for Imaging Science at Johns Hopkins University, Baltimore, MD. He is interested in developing sophisticated diffeomorphic registration approaches to study anatomical configurations. 